rev.relac.int.estrateg.segur.7(2):157-170,2012

\title{
NOO-POLÍTICA, EL GOBIERNO DE LA CONDUCTA DE LOS DEMÁS: UN ACERCAMIENTO AL PENSAMIENTO DE MAURICIO LAZZARATO*
}

\author{
Laity A. Velásquez F.*
}

\section{RESUMEN}

El presente trabajo circunscribe la mirada a los aspectos sobre política y vida que se encuentran en el filósofo y sociólogo italiano Mauricio Lazzarato. Se aborda la dinámica del acontecimiento que concibe el mundo como la coincidencia en conflicto de un doble devenir, valores dominantes y nuevas posibilidades. Pretende mostrar cómo a la luz de su ejercicio teórico, se puede encontrar una relación entre Bioética y Biopolítica, la acción política y la vida, sus posibilidades de modulación y resistencia.

Palabras clave: Biopolítica, Bioética, Acontecimiento, Política, Vida.

* Este trabajo de reflexión es producto de la acción académica ejercida en el Seminario de Biopolítica-Bioética que apoya la línea de Investigación del mismo nombre; producto del Proyecto de Investigación Bioética y Biopolítica: encuentros y desencuentros (HUM-946), del grupo de Investigación Bioethics Group en alianza con el grupo de Investigación Liderazgo, sociedad y bioética, ambos circunscritos al Doctorado de Bioética de la Facultad de Educación y Humanidades de la Universidad Militar Nueva Granada.

** Licenciada en Filosofía y Letras. Magister en Educación. Estudiante del doctorado de Bioética de la Universidad El Bosque.

Correo electrónico: laity.velasquez@unimilitar.edu.co 


\title{
NOO-POLITICS, THE GOVERNMENT OF THE BEHAVIOR OF OTHERS: AN APPROACH TO THE THOUGHT OF MAURICIO LAZZARATO
}

\begin{abstract}
This study directs our attention to aspects about political and life thoughts of the Italian philosopher and sociologist Maurice Lazzarato. This document explains the dynamic of an event which sees the world as coincidence in conflict, a double change of dominant values and new possibilities. It aims to show how under the light of his theoretical exercise, a relationship can be found between bioethics and biopolitics, political action and life, its modulation possibilities and resistance.
\end{abstract}

Key words: Biopolitics, Bioethics, Event, Politics, Life.

\section{NOO-POLÍTICA, O GOVERNO DA CONDUTA DOS DEMAIS: UMA APROXIMAÇÃO AO PENSAMENTO DE MAURÍCIO LAZZARATO}

\section{RESUMO}

Este trabalho circunscreve um olhar sobre as questões de política e vida presentes no filósofo e sociólogo italiano Maurice Lazzarato. Aborda-se a dinâmica do evento que vê o mundo como a coincidência em conflito com um duplo acontecer, valores dominantes e novas possibilidades. Pretende mostrar como, sob a luz do exercício teórico, é possível encontrar uma relação entre a Bioética e a Biopolítica, a ação política e a vida, suas capacidades de modulação e resistência.

Palavras-chave: Biopolítica, Bioética, Evento, Política, Vida

\section{INTRODUCCIÓN}

El presente trabajo circunscribe la mirada a los aspectos sobre política y vida que se encuentran en el filósofo y sociólogo italiano Mauricio Lazzarato. Con el ánimo de encontrar resonancias que permitan situar su ejercicio teórico a la luz de una perspectiva Bioética, se han desglosado siete acápites en los cuales se aborda tal problemática desde diferentes aristas. Así, pasando por la dinámica del acontecimiento y sus implicaciones éticas y políticas, llegaremos a una posible relación entre Bioética y Biopolítica en el marco de este importante recorrido.

La Biopolítica, que comprende las prácticas del gobierno de las conductas: cómo dirigir las conductas de los demás y cómo gobernarse a sí mismo, encuentra un desarrollo en la Noopolítica que se centra en el gobierno de los demás en las sociedades contemporáneas. Esta 
será la problemática abordada por Mauricio Lazzarato en su libro Políticas del acontecimiento. Su planteamiento aborda las relaciones de poder en las sociedades de control, como un poder a distancia que actúa sobre las conductas de los individuos. Tal como lo pretendiera M. Foucault (Lazzarato, 2006, p.11), "las técnicas de seguridad (o de control si se utiliza la definición de Deleuze) deben actuar sobre las reglas del juego, más que sobre el juego mismo". Es necesario señalar que establece una distancia de las concepciones que han explicado el mundo a partir de la relación sujeto/objeto, y se sitúa en la filosofía del acontecimiento que tiene otra mirada y hace posibles desarrollos distintos. Continúa la línea de pensamiento de $\mathrm{M}$. Foucault y G. Deleuze, que complementa con la revisión de autores como Leibniz, G. Tarde, Bergson, W. James, M Bajtin.

\section{LA ACCIÓN POLÍTICA EN LA LÓGICA DEL ACONTECIMIENTO}

Según Lazzarato (2006, p. 48), "el mundo es un posible que se actualiza en las almas y se encarna en los cuerpos, esta idea de Leibniz se rehace en el pensamiento de G. Deleuze, como el mundo es un virtual, una multiplicidad de relaciones y de acontecimientos que se expresan en un agenciamiento colectivo de enunciación (en las almas) que crean lo posible".

Entender la afirmación anterior implica pararse en otra esquina de las explicaciones y mirar el mundo desde el papel del paradigma de la subjetivización'. Para Felix Guattari (1996, p. 20), "hacer transitar las ciencias humanas y las ciencias sociales desde los paradigmas cientificista hacia paradigmas éticos-estéticos (...), cada individuo, cada grupo social vehiculiza su propio sistema de modelización de subjetividad es decir una cierta cartografía hecha de puntos de referencia cognitivos pero también míticos, rituales, sintomatológicos, a partir de los cuales cada uno se posiciona en relación con sus afectos, sus angustias e intenta administrar sus inhibiciones y pulsiones".

La coincidencia en el tiempo y el espacio de intensidades como fuerzas deseántes instituidas y futuras, permite identificar al acontecimiento. Lo posible que se piensa y se practica según la idea inicial, se expresa en dos regímenes de verdad: creación de lo posible/consumación y posible/realización. Estas duplas coexisten, se imbrican, no se sustituyen una a la otra, lo que induce a pensar el mundo como conflicto. Cada uno de los regímenes lleva a una alternativa; en el caso de lo posible/realidad conlleva la creación dentro de las condiciones de posibilidad dadas; en el segundo caso creación de lo posible/consumación, la alternativa es de superación de lo dado anteriormente. En esta dinámica podemos evidenciar el alcance de la noción de acontecimiento y sus posibilidades de resonancia en la comprensión de lo social.

1. Conjunto de condiciones por las que instancias individuales y/o colectivas son capaces de emerger como territorio existencial sui-referencial, en adyacencia o en relación de delimitación con una alteridad a su vez subjetiva. 
La acción política es una creación doble que a la vez recibe la nueva distribución de los posibles y trabaja para su consumación en las instituciones, en los agenciamientos colectivos ${ }^{2}$. De la misma manera el movimiento que se efectúa es precisamente en palabras del autor una efectuación de mundos, no trasformación sino desplazamiento que sitúa frente a enunciados colectivos diferentes, actualización en las almas de mundos posibles. Lo político comporta estos dos aspectos.

Esto podría constituir un cambio radical en relación con la tradición del movimiento obrero, ya que el acontecimiento político define una asimetría en la dialéctica con la cual se ha aprehendido, siguiendo al marxismo, el conflicto y la lucha. El "no" dirigido al poder, no es más que el punto de partida de una lucha dialéctica contra él, sino la apertura a un devenir. Decir "no" constituye la forma mínima de resistencia. Esta última debe abrir un proceso de creación, de trasformación de la situación, de participación activa en el proceso. Esto es resistir según Foucault (Lazzarato, 2006, p. 50)

Es así como un acontecimiento político es un momento y un espacio que comporta el movimiento de los dos aspectos: el "no" con lo existente, en la esfera de (posible/realización) y una primera fase de creación de lo posible, sólo en lo que respecta a la actualización en las almas de los enunciados, es decir en la dupla corresponde a creación de lo posible. Pero como es necesario efectuarlos, consumarlos, ya que en el primer sentido, se ha dado lo que posibilita un cambio en la manera de sentir, ha cambiado la distribución de los deseos, a la luz de esta nueva luz del entendimiento, se ve lo intolerable y simultáneamente las nuevas posibilidades de vida, pero aún falta la consumación, realización o efectuación.

Refiriéndose a lo ocurrido en las Jornadas de Seattle ${ }^{3}$ Lazzarato, 2006, p. 52) dice: "únicamente el acontecimiento crea la posibilidad de un nuevo objeto (una nueva política-mundo, un nuevo transnacionalismo) y la posibilidad de un nuevo sujeto (que no es más la clase obrera, pero que no es todavía más que una multiplicidad posible)". De la misma manera en la posición de los nuevos socialismos, se plantea precisamente esta bifurcación como necesaria, subordinan la acción a la creación de una bifurcación, de una desviación, de un estado inestable que al suspender y neutralizar las oposiciones binarias [dadas por el régimen de lo posible/realización], abren un nuevo campo de lo posible.

2. El termino colectivo ha de entenderse aquí en el sentido de una multiplicidad que se despliega a la vez más allá del individuo, del lado del socius y más acá de la persona, del lado de las intensidades pre-verbales tributarias de una lógica de los afectos más que de una lógica de conjuntos bien circunscritos.

3. A finales de noviembre de ese año [1999] tuvieron lugar las movilizaciones en Seattle, durante el Encuentro Ministerial de la OMC de la llamada Ronda del Milenio, durante cinco días que marcaron "un antes y un después" en la trayectoria del movimiento. En Seattle confluyó un amplio espectro de organizaciones y redes de diferentes países cuyo centro de actividad era desde hacía tiempo la enuncia de la OMC y sus proyectos liberalizadores, así como diversos de movimientos de Estados Unidos". 


\section{LA DINÁMICA DEL ACONTECIMIENTO}

Si no hay sujeto y objeto es factible explicar la constitución del mundo y del yo. Ante esto, ¿qué plantea la filosofía del acontecimiento? El concepto de mónada sirve para mirar este proceso; en Leibniz son fuerzas constitutivas de deseo, creencias, percepción, memoria, el individuo y todo el universo está constituido por mónadas, y podemos derivar la idea de un universo vivo. De tal manera que el mundo y lo individual es un conjunto de relaciones, físicas, vitales, sociales que se combinan. En Leibniz, las mónadas actúan y se coordinan mediante una "armonía preestablecida", un acuerdo universal, garantizado por Dios. En la filosofía del acontecimiento, el concepto de captura se refiere a la coordinación entre las mónadas: "se puede decir que una mónada actúa, es decir modifica la manera de sentir de otra mónada, poseyéndola, capturándola" (Lazzarato, 2006, p. 62).

Leibniz y las explicaciones a partir de la relación sujeto/objeto coinciden en el planteamiento del mundo actual, como el único posible existencialmente, ya que para Leibniz, todos los mundos posibles existen en el entendimiento de Dios, y son incomponibles (no coincidentes) con el actual, pero no son imposibles. De tal manera que el mundo donde Adán pecó (el nuestro) puede tener su contrario, un mundo donde Adán no pecó y existir en mundos diferentes, que son incomponibles uno con el otro. Este planteamiento es ampliado por G. Tarde que considera que todos los mundos incomponibles pueden pasar a la existencia al mismo tiempo, ya que "como recalca Deleuze, esto sería globalmente posible, ya que la incomponibilidad es una relación original distinta de la posibilidad y la contradicción" (Lazzarato, 2006, p. 64). Esta mirada permite al mundo contemporáneo (para el autor el mundo posterior a la muerte de Dios que ha dejado a las mónadas sin armonía preestablecida y frente a infinidad de mundos posibles), tener la doble oportunidad de hacer la selección de la serie de mónadas y armonizar su relación, y crear una infinidad de mundos. De esta manera no tenemos por qué plantear un principio social fuera de la acción constitutiva e inmanente de todas las mónadas. Pensar el mundo puede ser una tarea en la que no tengamos que recurrir a las categorías duales de naturaleza/sociedad, sujeto/objeto, individuo/colectivo, micro/macro.

\section{LO SOCIAL, ¿QUÉ ES?}

La pregunta realizada sobre ¿cómo utiliza G. Tarde la lógica del acontecimiento de Leibniz en la explicación acerca del funcionamiento de la sociedad?, pasa por establecer un primer elemento: en la filosofía del acontecimiento, la sociedad es el conjunto de las singularidades, la visión tiene origen en la concepción leibniziana del socius o mundo social, sobre la base de la dinámica que imprime la dupla creación de lo posible/consumación o propagación. Efectivamente, esta dinámica es semejante a un cerebro social, donde los cerebros particulares son las células (singularidades) que a partir de la cooperación (coordinación y acción) actúan unas sobre otras, a través de la acción a distancia de los deseos y las creencias. Aquí, cabe destacar que los flujos 
de deseos no dependen de los cerebros que desean, ellos no los crean, sino que son fuerzas, corrientes de imitación o corrientes de invención. Un segundo elemento es sobre la concepción del cerebro social, como una metáfora para referirse al funcionamiento del socius, pero no para describir su conformación o estructura, de tal manera que la sociedad no es un todo con partes, sino una multiplicidad.

Ya hemos afirmado los dos movimientos de la dinámica de lo social, uno que es conducido por las corrientes de imitación o afectación y otro que es conducido por las corrientes de invención. Es decir, estamos hablando acerca del papel de la acción de las singularidades, en la reproducción o cambio del todo o en otras palabras en la creación de lo posible/consumación o propagación. En lo que respecta al primer elemento de la dupla, este proceso es una dinámica doble. Por un lado el proceso de creación es producto de las corrientes de invención, que tiene la particularidad de producir asociaciones, de combinar deseos, para hacer emerger una nueva potencia y surgir como ex abrupto, de repente en un espíritu. En cuanto al segundo elemento (consumación/propagación), el conjunto de redes constituidas, reciben la invención que es asimilada, convertida en hábito, en cuerpo, pero tiene la posibilidad de bifurcarse, de denegarse, para crear nuevos posibles. Esto es lo que entiende G. Tarde por creación de Valor (invencióndifusión) y cooperación que también se encuentran en el pensamiento de Deleuze.

La invención implica, pues, un doble proceso de desubjetivación que abre una nueva producción de subjetividad, que concierne tanto a la singularidad que produce algo nuevo como al público que participa en esta co-creación y la prolonga ya que ambos deben escapar a los hábitos establecidos (a lo social) a las alternativas binarias que imponen (...) A diferencia del trabajo [la categoría marxista de trabajo, que ha venido presentado como un muestra de las filosofías del sujeto/objeto] esta acción también es inmediatamente pública, ya que está abierta a todos; se hace bajo los ojos, los afectos, las inteligencias y las voluntades de todos. Esta publicidad abre al encuentro acontecimental de los posibles y no al reconocimiento de lo subjetivo (Lazzarato, 2006, p. 70).

En esta parte se señala el otro elemento de la dupla que corresponde a la consumación o efectuación. Es precisamente las relaciones que establecen las mónadas entre sí (captura y apropiación) que hace que entre ellas se establezcan fuerzas de cohesión, organizaciones en redes, en agregados, en mallas, en tejidos en que fluyen los deseos o las creencias. En este entramado, no se pierde la singularidad, se co-actúa en relaciones conjuntivas o disyuntivas (inclusivas o exclusivas). Allí puede tener lugar la puesta en común de la diferencia o invención, y dado que funciona en coordinación, no se subsumen en una totalidad, se distribuyen; según Deleuze, los todos distributivos, al respecto, se reflejan a través de "la coordinación, el ser conjunto, (que) expresa una potencia en la cual las mónadas, las singularidades existen una a una, cada una por su propia cuenta (...), está es la naturaleza de las cosas. La afirmación de un modo de constitución en proceso y en archipiélago" (Lazzarato, 2006, p. 71- 72). 


\section{LOS AGREGADOS Y LA VIDA}

El ritornelo no es la coronación de la obra de la naturaleza en la forma consumada del sujeto o de la comunidad, sino el lugar mismo de una subjetivación imposible [producto de coordinación de conjunciones y disyunciones]. El proceso de subjetivación siempre es una jerarquización momentánea, una clausura provisoria de una multiplicidad de fuerzas, que supone a la vez la organización de una cooperación y el mando de esa cooperación. El individuo (célula, ser humano o sociedad) se constituye en la distancia entre la acción del principio coordinador y la voluntad de apropiación del mundo, es decir, como movimiento continuo para superar esta coordinación (Lazzataro, 2006, p. 77).

Pareciera algo obvio, el significado de la palabra vida, según la afirmación anterior. La singularidad implícita en el proceso, la ubica como un acto de invención, una fuga. Sin embargo, su acepción como Bíos, desde que Aristóteles la sitúa en la polis, la inscribe en el campo de la política. Por ello, la vida no se separa de su forma, la política o lo que es lo mismo, forma de vida o Bíos. Para comprender esta categoría en su dinámica actual, nos sitúa Lazzarato en la polis moderna: las sociedades de control, y su modulación del Bios, explicación que encuentra en Deleuze. Es necesario para ello apartarse del mismo Deleuze, para dialogar con Foucault en el tránsito de las sociedades disciplinarias a las de control y de paso establecer distancia con el marxismo. De la misma manera, remitirse a Nietzsche, Tarde y Bergson en la noción de memoria.

Para Lazzarato, M. Foucault, que habla siempre de relaciones de poder, apunta a una concepción del poder como y sobre la multiplicidad (Foucault, 2010a). En este sentido se aparta de las categorías marxistas de capital/trabajo como única lógica del poder y de la ideología como subjetivización, para integrar un marco más amplio, el de las sociedades disciplinarias y su doble técnica del poder: disciplinas y biopoder. En una u otra el poder que en las disciplinas se ejerce sobre el cuerpo, la multiplicidad es encerrada, cuadriculada, puesta en serie, es decir ordenada en el espacio-tiempo para extraer de ella efectos útiles, aumentando su producción. En cuanto al ejercicio del poder en la Biopolítica (Foucault, 2010b), se gestiona la población, la multiplicidad es numerosa y el espacio es abierto. Por otra parte, desde la mirada de Deleuze el análisis de Foucault remite a las categorías de diferencia y repetición, que pone al poder como relación de fuerzas y a las instituciones como agentes de integración.

Las relaciones de poder son virtuales, inestables, no localizables, no estratificadas, potenciales, y definen solamente las posibilidades, las probabilidades de interacción; las relaciones diferenciales determinan las singularidades. La actuación de estas relaciones diferenciales de esas singularidades, por parte de las instituciones (Estado, Capital etc.,) que las estabilizan las estratifican, que las hacen no reversibles es a la vez integración (captura) y una diferenciación (Foucault, 2010b). 
Sin que el autor lo mencione de esta manera, podríamos entender que en el proceso de integración y diferencia existe la semejanza entre Foucault y Deleuze, ya que en el planteamiento de las relaciones de poder y su actualización están presentes los procesos de integración y diferenciación. El concepto de integración (Lazzarato, 2006, p. 85) remite a "una operación que consiste en trazar una línea de fuerza general que pasa por las fuerzas y las fija en formas (...) la actualización de las relaciones de poder se hace poco a poco (...) es un conjunto de integraciones, primero locales y después globales. Deleuze describe la integración como un procedimiento para hacer que se mantengan juntos los networks y los patchworks, los flujos y los agregados". En cuanto a la diferenciación, es necesaria para el ejercicio del poder. Sin embargo, en el análisis de Foucault que apunta al capitalismo, el ejercicio del poder se manifiesta como represión de la variabilidad de la diferencia, mirada como reproducción y producción; Lazzarato (2006, p. 85), afirma que "en el capitalismo, esta diferenciación, en lugar de ser diferenciación de la diferencia, despliegue de la multiplicidad, es una creación y una reproducción de dualismos, los cuales los más importantes son los dualismos de clase (proletarios/capitalistas) y los dualismos de sexo (hombres/mujeres)". Sigue afirmando que las dos modalidades del ejercicio del poder no son contradictorias ${ }^{4}$, por lo que para dar salida al economicismo y a los dualismos, Foucault plantea que una sociedad la definen los enunciados que la expresan y las visibilidades que se efectúan; en Deleuze y Guattari serán máquinas de expresión y agenciamiento corporal.

Sin embargo, el planteamiento de Foucault va más allá de él mismo,

Las instituciones disciplinarias (...) operan una representación más profunda, no porque nieguen una naturaleza humana que ya está ahí, sino porque las disciplinas y el biopoder separan las diferencias de la dinámica "diferencia que va difiriendo". Disciplinas y biopoder son modos de producción de la subjetividad, pero únicamente cuando la infinidad de la monstruosidad que contiene virtualmente el alma (el devenir del monstruo) ya ha sido sometida a los dualismos (hombre/mujer, patrón/obrero etc.) (Lazzarato, 2006, p. 87).

En cambio para Deleuze, el monstruo se despliega aquí y ahora, como modalidad de subjetivización, y es lo característico de las sociedades contemporáneas, a partir de la identificación del mundo actual como una proliferación de mundos posibles ya anunciada por G. Tarde. Según Lazzarato (2006, p. 89), "lo que ocurre a finales del siglo XX: que esta voluntad de encerrar el afuera de hacer pasar a la existencia un solo mundo disciplinado entre la infinidad de los mundos posibles ha fracasado (...) la mónadas huyeron del mundo disciplinado inventando mundos incomponibles que se actualizan en el mismo mundo"; se presenta así para Deleuze,

4. En su nota (7) del escrito dice: La diferencia de punto de vista entre Foucault y Deleuze sobre la acción y la eficacia de la represión se debe a la preeminencia que éste último da, a diferencia de Foucault, al agenciamiento de deseo sobre el agenciamiento de poder. 
un cambio radical de las formas de organización y ejercicio del poder: "el agenciamiento de la diferencia y de la repetición ya no puede ser neutralizado, sino que debe ser controlado en tanto que tal" (Lazzarato, 2006 p. 90); la dinámica es ahora de control en un espacio abierto, es decir modulación.

El concepto de lo público, que Deleuze toma de G. Tarde sirve para mostrar el paso de las sociedades disciplinarias a las sociedades de control. "El público es una masa dispersa donde la influencia de los espíritus de unos sobre otros se convierte en una acción a distancia" (Tarde, 1986). Este proceso va de la mano con los desarrollos de las sociedades actuales, tales como:

(1) La emergencia de la cooperación entre cerebros y su funcionamiento por flujos y por redes; (2) el desarrollo de los dispositivos tecnológicos de acción a distancia de las mónadas: teléfono, cine, TV, net etc.; (3) los procesos de subjetivización y de sometimiento correspondientes, la formación de los públicos, es decir el ser conjunto que tiene lugar en el tiempo. (Lazzarato, 2006, p.92).

La máquina de expresión social y tecnológica es diferente y ella se convierte en un lugar estratégico para la actualización del acontecimiento en las almas. Allí tiene lugar la integración y diferenciación de las relaciones de poder, nacen unas nuevas técnicas (la acción a distancia) y unas nuevas instituciones (la opinión pública, la percepción colectiva y la inteligencia colectiva).

Esto constituye el carácter y la eficacia de la acción del público, en ser elástica en la medida en que los individuos no establecen una relación de pertenencia exclusiva, ni de identidad, se hacen relaciones múltiples y miméticas,

Hacen que el reino de moda reemplace al de la costumbre, o la innovación a la tradición, el control de la opinión, del lenguaje, de los regímenes de signos, de la circulación de los saberes, del consumo etcétera, remite a técnicas de poder inéditas que serán descritas después por Tarde, por el trabajo de Bajtin en la Rusia Soviética de los 20 y por la filosofía de Deleuze y Guattari alrededor de 1968. (Lazzarato, 2006, p. 94).

\section{LA VIDA Y LO VIVO}

Es ahora el concepto de memoria, inserto en la potencia de actuación de lo virtual, que servirá para mostrar el ejercicio del poder que se ejerce en las sociedades de control, pero desde una nueva dinámica abierta y coincidente con las dinámicas de singularización y movimiento del público. Lo vivo es memoria, Nietzsche y Tarde se encuentran con esta definición a partir de los desarrollos de científico Haeckel, 
Para el biólogo alemán todos los elementos infinitesimales (plasticidades) de un cuerpo, todas las mónadas orgánicas tienen una memoria, en tanto que esta propiedad (o aptitud) le falta al no-vivo" (Lazzarato, 2006, p.97). [Así mismo es una constante que se expresa en los estudios de biología y fisiología contemporánea], la esencia de lo vivo es una memoria, la preservación física del pasado en el presente. Al reproducirse las formas de vida vinculan el pasado al presente y graban los mensajes para el futuro. (Lazzarato, 2006, p. 97).

La memoria preserva el antes en el después; para Bergson, discípulo de Tarde, es una duración que comporta una actividad de actualización y repetición. Esta actividad (atención), es un esfuerzo o trabajo intelectual, para Bergson y conatus para Tarde. Según (Lazzarato, 2006, p. 98) "recordar algo y toda la actividad del espíritu en general, es actualizar un virtual y esta actualización es una creación, una individuación y no una simple reproducción". Con más precisión, la atención o trabajo intelectual, o actividad o esfuerzo, puede mirarse como un deseo. Las tecnologías de acción a distancia, son agenciamiento maquínico de enunciación que modulan (ejercicio del control, en Deleuze) las fuerzas de la memoria - atención y las relaciones mediante las cuales se actualizan, y los flujos de deseos y creencias.

Tenemos entonces una nueva modalidad de ejercicio del poder mediante la modulación y una distinción entre el Bíos que señala el biopoder (cuerpos y poblaciones) y el Bíos como memoria. Así mismo, la instauración de un nuevo campo de conocimiento, la Noo-política como,

El conjunto de técnicas de control [que] se ejerce sobre el cerebro, implicando en principio la atención, para controlar la memoria y su potencia virtual (...) la modulación de la memoria constituye la función más importante de la noo-política (...) las sociedades de control modulan los cerebros y constituyen hábitos especialmente en la memoria espiritual. (Lazzarato, 2006, p.100).

El anterior señalamiento del nacimiento de la Noo-política no invalida la coexistencia que el ejercicio del poder implica en las sociedades actuales, sino que señala lo que es inminente para el ahora; los tres dispositivos de poder no se sustituyen sino que se agencian unos a otros. La diferencia estriba en el grado de desterritorialización; la Noopolítica dirige y organiza los otros dispositivos, mientras que opera en el nivel más desterritorializado. La coexistencia de Bíos apunta a,

Un moldeado de cuerpos, asegurado por las disciplinas (prisiones, escuelas, fábricas, etc); a la gestión de la vida, organizado por el biopoder (Estado de Bienestar, políticas de salud etc.,): a la modulación de la memoria de las potencias virtuales regulada por la noo-política (redes hertzianas, audiovisuales, telemáticas y constitución de la opinión pública, de la percepción y de la inteligencia colectiva). Sociológicamente tendríamos esta secuencia: clase obrera (como una modalidad de encierro), la población y los públicos. (Lazzarato, 2006, p. 100-101). 


\section{FORMAS DE ASOCIACIÓN Y RESISTENCIA}

Pensar el mundo en la lógica del acontecimiento es considerar al otro como la expresión de mundos posibles y las relaciones de poder como capturas de flujos deseántes. M. Foucault distinguió como constitutivo de las relaciones de poder, las relaciones estratégicas, las técnicas de gobierno y los estados de dominación. Las posibilidades de asociación o de resistencia estarán relacionadas con las técnicas de gobierno, ya que permiten que los juegos estratégicos sean cerrados o abiertos, o se fijen en relaciones asimétricas o de dominación. Para Lazzarato, le corresponde a la acción política entenderse como una técnica de gobierno de sí y de los demás, un lugar de ejercicio de la libertad donde se experimente y trasforme, donde se impide el cierre de los estados de dominación dando cabida a la creación de los posibles y se hagan reversibles los juegos estratégicos a través de instituciones móviles o nómadas.

Este espacio entre la microfísica del poder y las instituciones de dominación (espacio que no está dado sino que hace falta inventar, construir y mantener) el propicio para una política del devenir y de la creación y para la invención de nuevas formas de subjetivización. Foucault, Deleuze y Guattari nos dicen que al fin de cuentas si se quiere pensar y practicar la política de la multiplicidad, hay que partir de estos espacios, de estas líneas trazadas cada vez de manera singular entre lo molar y lo molecular entre las relaciones de dominación y las relaciones estratégicas. Esto es exactamente lo que hacen los movimientos y lo que rechazan las instituciones molares (de derecha o de izquierda). Y el único medio para construir relaciones sociales que tengan un horizonte distinto a la guerra. (Lazzarato, 2006, p. 227).

\section{BIOÉTICA Y BIOPOLÍTICA}

¿Cómo podrían usarse las nociones de política y de vida que se han hecho visibles en el discurso de Lazzarato como una herramienta enriquecedora de una perspectiva bioética? La invitación de este acápite será intentar mostrar qué aporta la noción de vida y política en la crítica al capitalismo contemporáneo, a los discursos bioéticos actuales. Desde el nacimiento de la Biopolítica, en que M. Foucault sitúa el análisis en la vida y lo liga al cuerpo, la salud, la higiene, la sexualidad, los modos de relación y conducta con el propio cuerpo, se suscita un nuevo panorama para pensar la vida misma, no sólo como un término médico-científico y por lo tanto muy propio de la tradición Bioética, sobre todo en sus fundamentos biomédicos, sino desde otras perspectivas.

El hombre, definido en su ser biológico, tendrá que ser sistemáticamente diferenciado del animal y de la mera vida orgánica cuya continuidad amenaza toda definición estable e histórica de la humanidad o de la naturaleza humana. En todo caso, esa vida que en la formulación foucaultiana del biopoder emerge como legitimización y 
objeto de la modernidad política en su doble articulación entre el individuo y la población, emerge también como instauración de lo monstruoso, lo animalizado, lo impersonal, lo inhumano, como fuerza que atraviesa las construcciones del individuo y de lo humano y que las amenaza con su pura potencia de devenir y de alteración (Giorgi \& Rodríguez, 2007, p.11).

El desafío es continuado por G Deleuze, que desliga la vida de toda cualificación, de toda personalización, de lo humano y la sitúa en el campo de las fuerzas, como acontecimiento. "Uno es el índice de una multiplicidad: un acontecimiento, una singularidad, una vida...Una vida sólo contiene entidades virtuales, está hecha de virtualidades, de acontecimientos, singularidades" (Deleuze, 2007, p. 39-40). Para Agamben (2007, p. 81), el plano de inmanencia de Deleuze funciona como "un principio de indeterminación virtual en que el vegetal y el animal el adentro y el afuera, y hasta lo orgánico y lo inorgánico se neutralizan y transitan el uno hacia el otro". La inmanencia, que significa agente y paciente al mismo tiempo, constituye un movimiento de autoconstrucción o conatus: "Espinosa [lo define] como vida..., "la vida es la fuerza con que una cosa persevera en el propio ser" (Agamben, 2007, p. 87). Cuando Deleuze escribe que la vida es el campo de inmanencia variable del deseo, da entonces una definición spinoziana. Este planteamiento conlleva a pensar la constitución del mundo, sin un principio antropomórfico, al mismo hombre como la coordinación de diferentes relaciones físicas, vitales, fuerzas, nómadas, como agregados; se trata de dar autonomía e independencia a todos los seres. Se pierde la distinción entre naturaleza y sociedad, entre humano y no humano, solamente coordinaciones de nómadas, agregados.

Para la Bioética, desde la aspiración de R. Potter ${ }^{5}$, según G. Hottois (2011), aquellos que la han pensado como una propuesta de encuentro para la discusión de las grandes problemáticas de la vida; la propuesta Biopolítica (Hottois, 2011) constituye el confluir en una serie de preocupaciones o asuntos familiares, en el sentido en que abordan el mismo objeto y lo sitúan en el contexto contemporáneo. De alguna manera ambas temáticas pueden verse ligadas a la complejidad de la cultura, el desarrollo científico técnico y la responsabilidad ante el futuro.

5. Van R. Potter. Se le considera el acuñador del término Bioética, un derivado de la unión de dos palabras griegas, Bios -vida- y Ethos -carácter, ética-. Esta paternidad se le atribuye porque la primera vez que apareció el término Bioética en una publicación fue en un artículo suyo de 1970, titulado "Bioethics: The science of survival" -Bioética: La ciencia de la supervivencia-. En su libro de 1971 Bioethics: Bridge to the future -Bioética: Puente hacia el futuro- vuelve a aparecer, confirmando su paternidad. Al acuñar este término, Potter realizaba una propuesta de fundar una disciplina que integrase la Biología, la Ecología, la Medicina y los valores humanos. Mediante la mezcla terminológica de Ética y Biología fundó una sugerente imagen, aunque los contenidos de lo que defendía en sus libros tenían más que ver con la Biología y el cuidado del medio ambiente que con la práctica médica hacia la que se ha orientado la Bioética en la actualidad. Así, en su obra de 1971 ya citada, hablaba de la creación de "puentes" que permitieran al hombre sobrevivir ante las amenazas principalmente ambientales que implica el progreso técnico. Ya posteriormente, acentuó el sentido ecológico de su propuesta al acuñar en el año 1988 el sintagma "Bioética global". Texto en línea. Disponible en internet: http://www.bioeticas.org/bio.php?articulo52 
La mirada de G. Hottois es especialmente esclarecedora, si consideramos el papel que el desarrollo científico-técnico actual impone al mundo que no puede dejar de organizarse a su alrededor y nos coloca ante otras ontologías y otras subjetividades. Allí encontramos los planteamientos de M. Lazzarato quien como hemos señalado, se ocupa por el gobierno de los demás en las sociedades contemporáneas, donde las relaciones de poder se ejercen a distancia a través de un medio; este medio artificial donde fluye el poder característico solo de las sociedades actuales, se ha creado bajo el auge de las tecnologías comunicativas que caracterizan el mundo de hoy. La acción política entendida como coordinaciones, bajo la lógica del acontecimiento funciona en los dos planos, el de la resistencia o rechazo y el de la invención. Despliega una dinámica de subjetivización que es afirmación de la diferencia y composición de un común múltiple, no totalizante que se mantiene unido como un público. El acto de resistencia introduce discontinuidades, nuevos comienzos que afirman y establecen la proliferación de los mundos posibles.

¿Cómo impacta tal noción de vida y política en el discurso bioético? Indudablemente introduce otra dimensión a la discusión bioética, considerar la vida - en tanto que memoria - de la vida en tanto que características biológicas de la especie humana (muerte, nacimiento, salud, enfermedad, etc.) significa mirarla como vivencia, conexiones entre vivencias. Significa a su vez poner en relación dos discursos, en términos de lo que Paul Ricoeur en discusión con Jean Pierre Changeaux reflexiona y llaman el cuerpo objeto y el cuerpo sujeto (Changeux, J. P. \& Ricoeur, P., 2001).

\section{BIBLIOGRAFÍA}

- $\quad$ Agamben, G. (2007). La inmanencia absoluta (Vol. Ensayos sobre Biopolítica. Excesos de vida.). (G. \&. Giorgi, Ed.) Buenos Aires: Paidos.

- $\quad$ Antentas, J. M. (05 de Septiembre de 2011). VIENTO SUR. Recuperado el 05 de Septiembre de 2011, de http://www.vientosur.info/articulosabiertos/VS107_AtentasyVivas_ DeSeattlealacrisis.pdf.

- Changeux, J. P. \& Ricoeur, P. (2001). La naturaleza y la norma. México D.F.: Fondo de Cultura Económica.

- Deleuze, G. (2007). La inmanencia una vida... (Vol. Ensayos sobre Biopolítica. Excesos de vida.). (G. F. Rodríguez, Ed.) Buenos Aires: Paidos.

- Foucault, M. (2010a). La gubernamentalidad (Vols. En Obras esenciales. Vol. III: Estética, ética y hermenéutica.). Barcelona: Paidos. 
- $\quad$ Foucault, M. (2010b). El Nacimiento de la Biopolítica. Barcelona: Paidos.

- $\quad$ Foucault, M. (2010c). Obras esenciales. Barcelona: Editorial Paidos.

- Giorgi, G \& Rodríguez, F. . (2007). Ensayos sobre Biopolítica. Excesos de vida. (Compiladores, Ed.) Buenos Aires: Paidos.

- Guattari, F. (1996). Coosmosis. Buenos Aires: Ediciones Manantial.

- Hottois, G. (2011). Definir la bioética:retorno a los orígenes. (J. E. Triana, Ed.) Revista Colombiana de Bioética, 6(2), 62-85.

- Lazzarato, M. (2006). Políticas del acontecimiento. (P. E. Rodríguez, Trad.) Buenos Aires: Tinta Limón.

- $\quad$ Tarde, G. (1986). La opinión y la multitud. Madrid: Taurus. 